\title{
Biological and Molecular Characterization of a New Carlavirus Isolated from an Aconitum sp.
}

\author{
J. Cohen, M. Zeidan, A. Rosner, and A. Gera
}

First, third, and fourth authors: Department of Virology, Agricultural Research Organization, The Volcani Center, P.O. Box 6; and second author: The Plant Protection and Inspection Services, Ministry of Agriculture, Bet Dagan 50250, Israel.

Accepted for publication 27 December 1999.

\section{ABSTRACT}

Cohen, J., Zeidan, M., Rosner, A., and Gera, A. 2000. Biological and molecular characterization of a new carlavirus isolated from an Aconitum sp. Phytopathology 90:340-344.

A new virus was isolated from symptomless Aconitum napellus plants. The virus, for which the name Aconitum latent virus (AcLV) is proposed, has flexuous particles $640 \mathrm{~nm}$ in length. The experimental host range was limited to Nicotiana clevelandii. Electron microscopy studies of ultrathin sections of infected A. napellus tissues revealed the presence of elongated virus particles. No inclusion bodies characteristic of potyvirus infection were observed. AcLV was purified from naturally infected A. napellus by cesium chloride step gradient centrifugation. In sodium dodecyl sulfatepolyacrylamide gel electrophoresis of dissociated purified virus prepara- tions, a major protein component with a molecular mass of $35 \mathrm{kDa}$ was observed. Diagnostic antibodies that could specifically bind to virus particles were produced. The $5^{\prime}$ terminus (620 nucleotides) of the viral RNA was cloned and sequenced. It comprised 71 nucleotides from the untranslated $5^{\prime}$ terminus and 549 nucleotides of an open reading frame encoding 183 amino acids. Comparison of the predicted amino acid sequence with those of other plant viruses revealed 40 to $60 \%$ identity with several carlaviruses. Based on particle morphology, absence of inclusion bodies in ultrathin sections, the relative molecular weight of the coat protein, the nucleotide sequence, and predicted amino acid homology, it is suggested that this virus belongs to the carlavirus group.

Additional keywords: purification, serology.
Aconitum napellus (family Ranunculaceae) is a new geophyte attracting great demand in Europe as a cut flower. The crop, which is vegetatively propagated, was introduced into Israel in 1985 by importing tubers from the Netherlands. The growing area exceeded 15 acres; but among the factors that limit the expansion of the growing area are the contamination of the tubers with fungal diseases and the low natural propagation index. To the best of our knowledge, no viral diseases of Aconitum spp. have been reported.

During surveys of viral diseases conducted in Israel, Aconitum spp. plants grown in commercial farms were observed to exhibit mild mosaic symptoms. The causative agent was identified as $\mathrm{Cu}$ cumber mosaic virus (CMV) (5). During 1997, plants with unusual viral symptoms of mosaic and necrosis on leaves were observed. These plants were found to be doubly infected with CMV and an unidentified elongated filamentous virus. Here, we report on the purification and characterization of a new carlavirus referred to as Aconitum latent virus (AcLV) isolated from A. napellus. We also report the cloning and sequencing of the 5'-terminal region of the genome of this virus and its comparison to the published sequences of other carlaviruses.

\section{MATERIALS AND METHODS}

Virus source, transmission, and host range. Leaf samples of A. napellus grown in commercial screenhouses in Israel were examined for virus particles using electron microscopy (EM). Samples containing elongated particles were homogenized in $1 \% \mathrm{~K}_{2} \mathrm{HPO}_{4}$, and the sap was inoculated to Capsicum annuum, Chenopodium amaranticolor, Chenopodium murale, Chenopodium quinoa, $\mathrm{Cu}$ cumis sativus, Cucurbita pepo, Datura stramonium, Eustoma grandi-

Corresponding author: A. Gera; E-mail address: abedg@netvision.net.il

Publication no. P-2000-0217-01R

(C) 2000 The American Phytopathological Society florum, Gomphrena globosa, Hyoscyamus negro, Lycopersicon esculentum, Nicotiana benthamiana, $N$. clevelandii, N. glutinosa, $N$. rustica, N. sylvestis, N. tabacum cv. Samsun, N. tabacum cv. Samsun NN, N. tabacum cv. Xanthi nc, N. tabacum cv. White Burley, Petunia $\times$ hybrida, and Physalis floridensis predusted with Carborundum. These plants were maintained in the greenhouse and observed for symptom development.

The green peach aphid (Myzus persicae (Sulzer)) was used for transmission tests. A clone of $M$. persicae that originated from a single female was reared on healthy Japanese radish (Raphanus sativus) plants. Nonviruliferous apterous aphids were allowed to feed for 5 min either on A. napellus or on mechanically inoculated $N$. clevelandii. Five aphids were transferred to each of 10 healthy $N$. clevelandii plants and allowed to remain on the test plant for $12 \mathrm{~h}$. These plants were then sprayed with insecticide, maintained in the greenhouse, and observed for symptom development.

Virus purification. Leaves (100 g) of A. napellus, collected in the field, were homogenized in $200 \mathrm{ml}$ of $0.2 \mathrm{M}$ potassium phosphate, $\mathrm{pH} 7.6$, containing $3 \%$ ( $\mathrm{vol} / \mathrm{vol}$ ) urea and $200 \mu \mathrm{l}$ of thioglycolic acid for $4 \mathrm{~min}$ in an Omni-mixer (Sorval Inc., Newtown, CT). The virus was purified according to the method described by Zavriev et al. (19). For further purification, virus preparations were layered on cesium chloride step gradients (10 to $40 \%$ in $0.1 \mathrm{M}$ phosphate buffer, $\mathrm{pH} 7$, containing $20 \%$ sucrose) (2). The gradient columns were fractionated into $0.5-\mathrm{ml}$ fractions with the aid of a peristaltic pump, and the virus-containing fractions were checked by EM. Fractions containing the highest concentration of virus were pooled and centrifuged.

Virus concentration was estimated spectrophotometrically using an extinction coefficient of $2.1(\mathrm{mg} / \mathrm{ml})^{-1} \mathrm{~cm}^{-1}$ at $260 \mathrm{~nm}$ for carlaviruses (18).

Production of antibodies. Partially purified virus (ppV) suspensions of AcLV (about $0.1 \mathrm{mg} / \mathrm{ml}$ ) were emulsified with an equal volume of Freund's incomplete adjuvant and injected intramuscularly into both hip muscles of 6-week-old female New Zealand 
white rabbits as described previously (20). Alternatively, the coat protein $(\mathrm{CP})$ from purified virus preparations was denatured and separated by sodium dodecyl sulfate-polyacrylamide gel electrophoresis (SDSPAGE). Stained protein bands (SDS-PB) were excised from the gel and used for injection as described previously (7). Two weeks after the last injection, a booster injection with $\mathrm{ppV}$ was given. Antisera were collected 12 days after the last injection. These antisera were designated $\mathrm{ppV} / \mathrm{ppV}$ and SDS-PB/ppV, respectively. Antisera were tested by western blotting (16), enzyme-linked immunosorbent assay (ELISA) (4), and immunoelectron microscope (IEM) decoration tests as described by Milne and Luisoni (10).

Physicochemical properties. The relative molecular weight $\left(M_{\mathrm{r}}\right)$ of AcLV CP was determined by SDS-PAGE of purified virus preparation; $12 \%$ polyacrylamide gels $(75 \times 5 \mathrm{~mm})$ were prepared as described previously (7). The CPs of Potato virus $M$ (PVM) and Potato virus $S$ (PVS) were also analyzed. The PBs were either stained with $0.1 \%$ Coomassie brilliant blue or electroblotted to nitrocellulose (16) and probed with the polyclonal antiserum. Antibody binding was detected with anti-rabbit immunoglobulin G conjugated to alkaline phosphatase (BioMakor, Rehovot, Israel).

Crude plant extracts in $0.1 \mathrm{M}$ phosphate buffer or AcLV preparations were negatively stained with $20 \mathrm{~g}$ of uranyl acetate per liter and examined for virus in a JEOL 100CX II electron microscope (JEOL Ltd., Tokyo). Particle length was measured on $\times 50,000$ enlarged photographs, and the normal length was calculated from the measurements of 100 particles. For calibration of magnification, a cross-line grating replica with spacing of 2,160 lines per millimeter was used.

For ultrathin sections, segments $(2 \times 1 \mathrm{~mm})$ were excised from young leaves of $A$. napellus and fixed in $2.5 \%$ glutaraldehyde buffered with $75 \mathrm{mM}$ potassium phosphate, $\mathrm{pH} 7$, for $2 \mathrm{~h}$. The samples were then rinsed in the same buffer and postfixed in $1 \% \mathrm{OsO}_{4}$ for $3 \mathrm{~h}$. After a buffer rinse, the samples were dehydrated in a graded acetone series, embedded in an Epon-Araldite resin mixture, and polymerized as described by Orion and Franck (12). Sectioned material was stained with uranyl acetate followed by lead nitrate.

IEM decoration tests were conducted as described by Milne and Luisoni (10). For identification of the serological reactions of the virus with other carlaviruses, antisera against PVM and PVS, diluted 1:10 (provided by G. Loebenstein, ARO, Volcani Center, Bet Dagan, Israel) were tested. Homologous and heterologous decoration reactions were determined using antigen from crude extracts of infected plants.

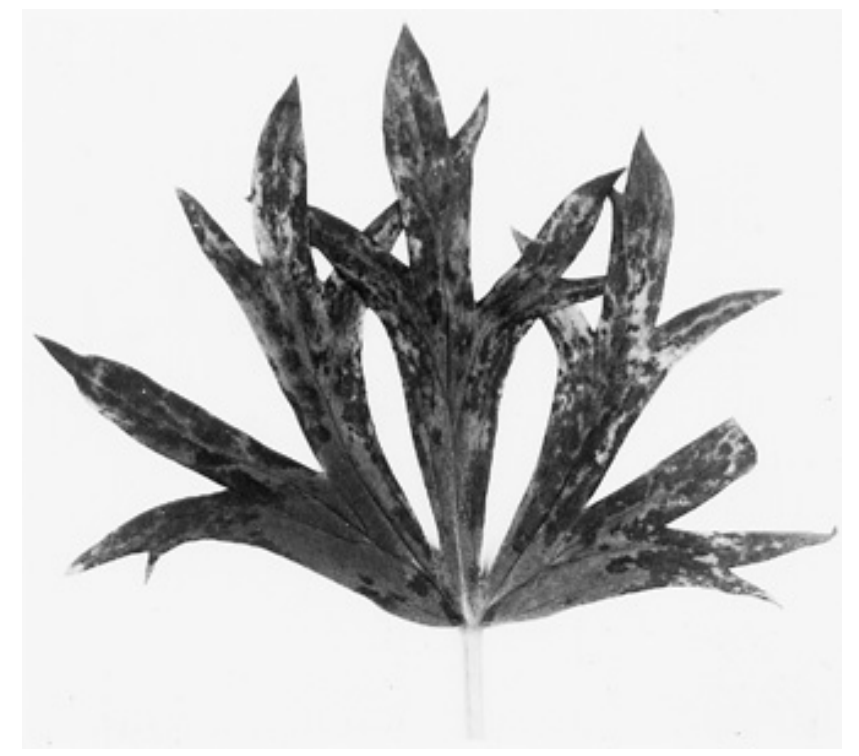

Fig. 1. Severe mosaic symptoms accompanied with necrosis on leaves of Aconitum napellus naturally doubly infected with Aconitum latent virus and $\mathrm{Cu}$ cumber mosaic virus.
cDNA synthesis and molecular cloning. RNA was extracted from purified AcLV particles by phenol/chloroform extraction as described previously (13).

The universal Riboclone cDNA Synthesis System Kit (Promega Corp., Madison, WI) with both random and oligo deoxyribosylthymine (dT) primers were used. cDNA synthesis was carried out according to the manufacturer's instructions. Double-stranded cDNA was blunted with T4 DNA polymerase and ligated into the SmaI site of a Bluescript plasmid vector using standard procedures (15). Escherichia coli DH5 $\alpha$ cells were transformed with the recombinant plasmid and plated on Luria-Bertani agar containing ampicillin, isopropyl- $\beta$-D-thiogalactopyranoside, and 5-bromo-4chloro-3-indolyl- $\beta$-D-galactopyranoside. White colonies were selected for plasmid analysis. Minipreps of plasmid were cleaved with $E c o$ RI and $\mathrm{XbaI}$ to screen for cDNA inserts by gel electrophoresis. Plasmid DNA of clones containing the insert was prepared by using the plasmid Midi-Kit (Qiagen Inc., Valencia, CA).

Sequence analysis. Selected recombinant plasmids were sequenced at the Weizmann Institute of Science, Rehovot, Israel, by the dideoxynucleotide chain termination method, using either forward or reverse M13 primers. Nucleotide and amino acid sequences were performed with the DNAMAN package, version 4 (Lynnon Biosoft, Quebec, Canada). AcLV-related sequences were initially identified using the BLASTP search program of GenBank (1). The sequence of the N-terminus 183 amino acids was compared with the following carlaviruses: Apple stem pitting virus, BAA04853 (8); Blueberry scorch virus, AAA68984 (3); Garlic latent virus, CAA92815 (C. Jin Nam, S. Sang Ik, S. Jong Tae, L. Jong Seob, and C. Yang Do, unpublished data); Grapevine rupestris stem pitting associated virus, AAC62910 (21); PVM, PN0093 (19); PVM Russian isolate, P17965 (19); Rupestris stem pitting associated virus, AAC35498 (9); and Sour cherry green ring mottle virus, AAC35433 (22).

Phylogenetic analysis was performed to determine the relationship of AcLV with other carlaviruses using the neighbor-joining algorithm in DNAMAN according to Saito and Nei (14). The data set was subjected to 1,000 bootstrap replicates. All nodes supported by $>45 \%$ confidence values are shown.

\section{RESULTS}

Virus transmission and host range. Initially, AcLV was detected in A. napellus plants that exhibited unusual viral symptoms of mosaic on leaves accompanied by necrosis (Fig. 1). These plants were found to be doubly infected with CMV and AcLV. In further tests, AcLV was detected in symptomless A. napellus plants. All A. napellus plants, representing the commercial stock grown in several different locations in Israel and tested by EM and ELISA, were found to be infected. Occasionally, mild green mottling in leaves developed when plants were exposed to high temperatures during the growing season.
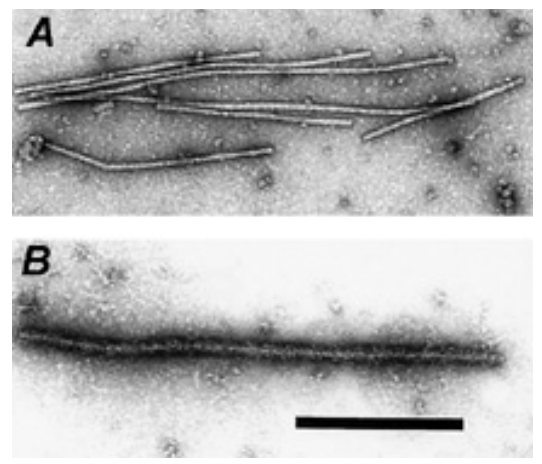

Fig. 2. Electron micrograph of purified Aconitum latent virus particles A, stained with uranyl acetate and $\mathbf{B}$, decorated with specific antiserum. Bar $=$ $250 \mathrm{~nm}$. 
AcLV was not mechanically transmitted to any of the 23 herbaceous plant species listed above, as determined by symptomology, for 1 month following mechanical inoculation. Tests by EM and ELISA confirmed occurrence of infection only in $N$. clevelandii. Attempts to transmit the virus mechanically from inoculated to healthy $N$. clevelandii were unsuccessful.

Transmission of AcLV by aphids from either naturally infected A. napellus or mechanically inoculated $N$. clevelandii to healthy $N$. clevelandii was unsuccessful.

EM. Flexuous, elongated virus particles were consistently observed in negatively stained samples from leaves of naturally infected $A$. napellus (Fig. 2A). The modal length of particles was about $640 \mathrm{~nm}$. The virus particles gave a distinct decoration with the specific antisera produced against the virus. Decoration tests

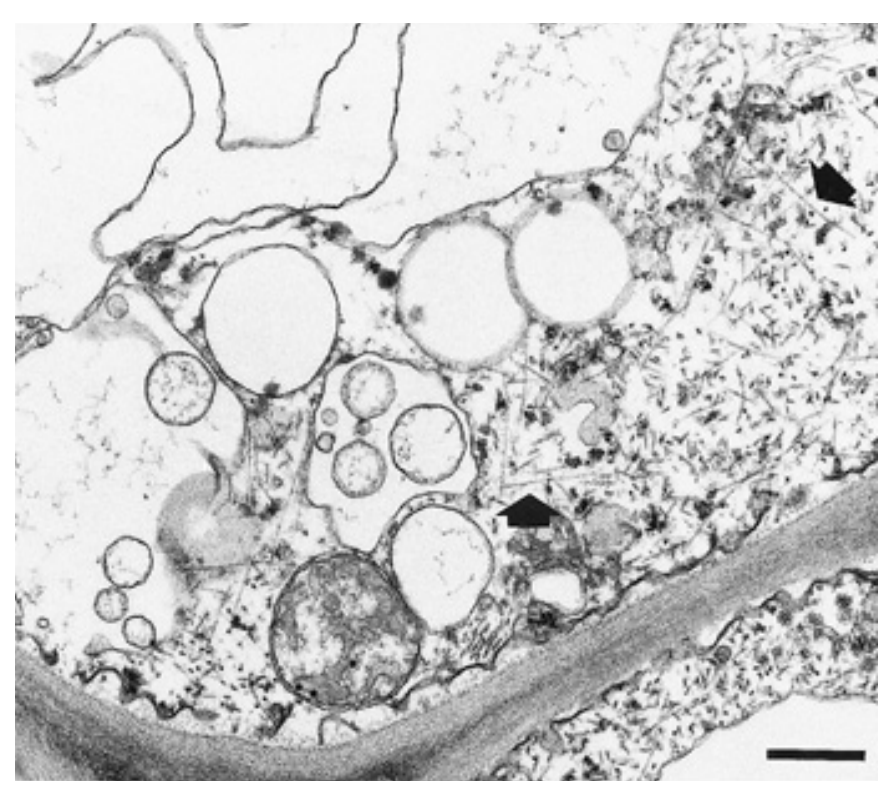

Fig. 3. Ultrathin section of Aconitum napellus leaf cells showing Aconitum latent virus particles in the cytoplasm (particles are indicated by arrows). Bar = $500 \mathrm{~nm}$.

with SDS-PB/ppV antisera resulted in a strong decoration of the particles (Fig. 2B).

EM studies using ultrathin sections of infected A. napellus tissues revealed the presence of flexuous, elongated rods resembling virus particles in the mesophyll, epidermal, and vascular cytoplasm of parenchyma cells (Fig. 3). Some of the chloroplasts in cells that contained these particles were vacuolated and disorganized. Cytoplasmic inclusion bodies characteristic of potyvirus infection were not observed.

Virus purification. Using the purification procedure outlined above, virus particles were located approximately $20 \mathrm{~mm}$ from the bottom of the cesium chloride gradient. Yields of purified virus from naturally infected A. napellus leaves ranged from 30 to $40 \mathrm{mg} / \mathrm{kg}$ of tissue. Preparations for EM of purified fractions contained large numbers of virus particles with relatively little contamination. The absorbance ratio of $260 \mathrm{~nm}$ to $280 \mathrm{~nm}\left(A_{260 / 280}\right)$ was found to be 1.235 , uncorrected for light scattering. The acceptable $A_{260 / 280}$ ratio for carlaviruses is 1.37 (18).

SDS-PAGE and serology. PAGE of SDS-disrupted purified virus preparations revealed one major and two minor polypeptide bands (Fig. 4A, lane 2). The $M_{\mathrm{r}}$ of the major protein was estimated to be $35,000 \mathrm{Da}$. This band was identified as the virus CP. It is uncertain whether minor bands were derived from virus particles or degradation products of the CP.

Immunoblots with SDS-PB/ppV and $\mathrm{ppV} / \mathrm{ppV}$ of AcLV gave a clear and strong reaction with the polypeptides corresponding to $\mathrm{CP}$, as identified by their $M_{\mathrm{r}}$ (Fig. 4B, lanes 3 and 4). Both AcLV

TABLE 1. Percent identity between the partial amino acid sequence of Aconitum latent virus open reading frame 1 and sequences of related carlaviruses

\begin{tabular}{lc}
\hline Virus & Amino acid identity (\%) \\
\hline Potato virus $M$ & 64.1 \\
Potato virus $M$ Russian isolate & 63.6 \\
Blueberry scorch virus & 47.8 \\
Grapevine rupestris stem pitting associated virus & 42.9 \\
Rupestris stem pitting associated virus & 42.9 \\
Garlic latent virus & 41.3 \\
Apple stem pitting virus & 37.1 \\
Sour cherry green ring mottle virus & 36.7 \\
\hline
\end{tabular}

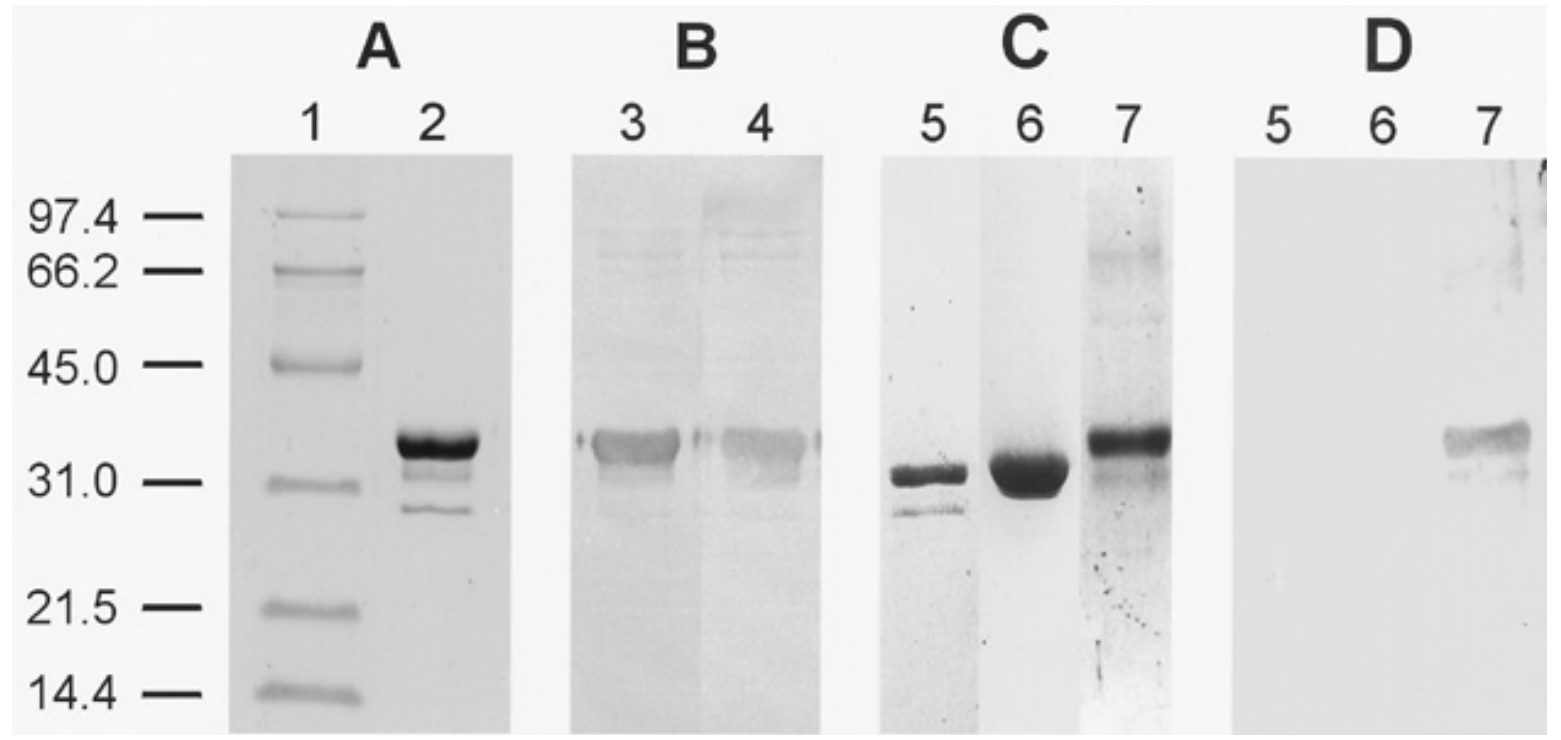

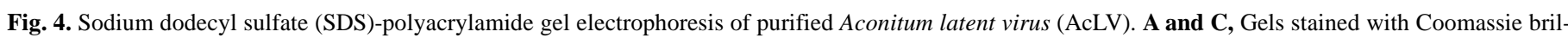

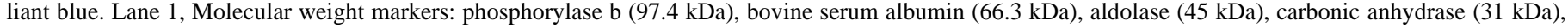

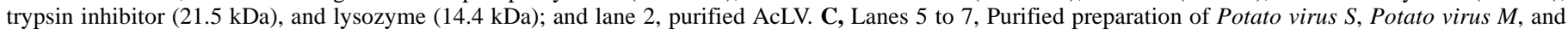

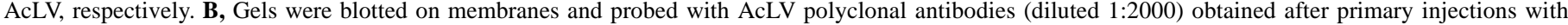

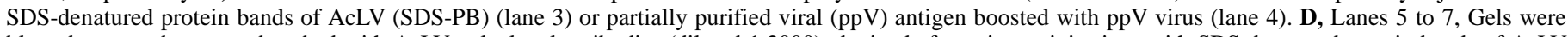

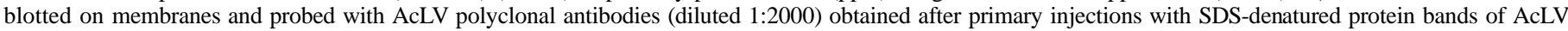
(SDS-PB). 
antisera specifically reacted with the $\mathrm{CP}$. In comparing the mobility of AcLV CP in SDS-PAGE with that of other carlaviruses, the estimated size of PVS, PVM, and AcLV CPs was found to be 33, 34 , and $35 \mathrm{kDa}$, respectively (Fig. 4C). In reciprocal tests with AcLV, PVS, and PVM antisera in immunoblots, only homologous reactions were obtained (Fig. 4D, AcLV).

In ELISA tests, considerably higher titers were obtained with the SDS-PB/ppV than with $\mathrm{ppV} / \mathrm{ppV}$ antisera (data not shown). The reactions of SDS-PB/ppV antisera were highly specific and more efficient in detecting AcLV, even when infected plant material was used at a dilution of 1:10,000, whereas the $\mathrm{ppV} / \mathrm{ppV}$ antisera failed to detect the virus at dilutions beyond 1:1,000 when ELISA was used.

Cloning the AcLV genome. DNA complementary to the 5'terminal region of AcLV was generated by using the universal Riboclone cDNA Synthesis System Kit with both random and oligo dT primers. A 620-bp clone derived from the 5'-terminal sequence of the viral RNA was selected for further study. This clone was sequenced, and the nucleotide sequence of the $5^{\prime}$ nontranslated region ( $\left.5^{\prime} \mathrm{NTR}\right)$ was established and deposited in the GenBank database under the accession number AF177225. It was comprised a 71-nucleotide, untranslated $5^{\prime}$ terminus and 549 nucleotides encoding 183 amino acids in open reading frame (ORF) 1. The sequence exhibited significant identity ( 40 to $60 \%$ ) to the N-terminal sequences of other carlaviruses (Table 1). The relatedness of AcLV to other carlaviruses based on partial polypeptide sequence homology is shown in a phylogenetic tree (Fig. 5). These results suggest that AcLV shares high identity with PVM and that both are clearly distinct from other carlaviruses.

\section{DISCUSSION}

AcLV seems to be similar to other carlaviruses in particle length and morphology, the apparent $M_{\mathrm{r}}$ of the $\mathrm{CP}$, and the nucleotide and the predicted amino acid sequences of the $5^{\prime}$ terminus. Like many carlaviruses, AcLV is symptomless in its infected natural host and has a narrow host range. The cytological properties of the virus are apparently different from those of potyviruses, and cytoplasmic inclusions typical of potyviruses were not found in infected Aconitum spp. (18).

All attempts to find alternative propagation hosts for AcLV failed. Although, the virus was transmitted to $N$. clevelandii from A. napellus, further attempts to passage the virus mechanically or by aphids to more healthy $N$. clevelandii were unsuccessful.

\subsection{5}

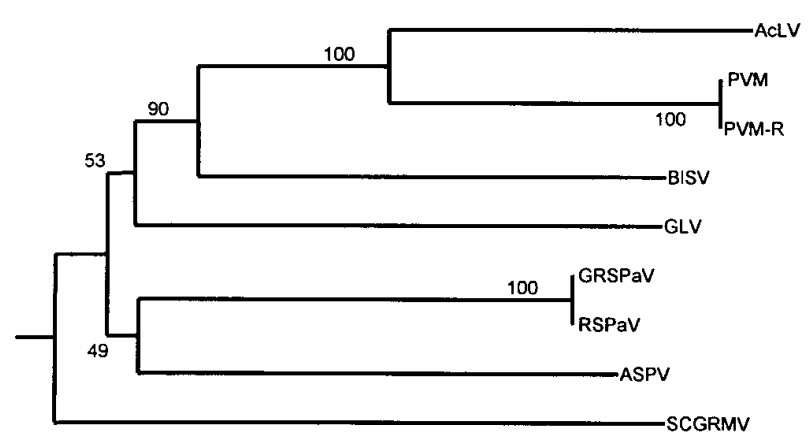

Fig. 5. Relationship of Aconitum latent virus (AcLV) with selected carlaviruses. The deduced amino acid sequence of open reading frame 1 was compared first by a BLASTP search of GenBank. Tree descriptions were generated using the neighbor-joining algorithm and based on calculations from pairwise amino acid sequence distances derived from the multiple alignment format (data not shown). Horizontal scale indicates sequence divergence and vertical scale is arbitrary. The data set was subjected to 1,000 bootstrap replicates. All nodes supported by $>45 \%$ confidence values are shown.
AcLV can be purified directly from A. napellus leaves. Virus preparations purified by this procedure were infectious in $N$. clevelandii by mechanical inoculation. The $M_{\mathrm{r}}$ of $35 \mathrm{kDa}$ observed in SDS-PAGE for the CP was in agreement with those published for other carlaviruses, but different in size from the CPs of PVS (33 kDa) and PVM $(34 \mathrm{kDa}),(18,19)$. The two minor PBs observed may be a breakdown product of the CP. Serological reaction in western blot and decoration test with SDS-PB/ppV and $\mathrm{ppV} / \mathrm{ppV}$ of AcLV were very specific and gave clear and strong reactions with the polypeptide corresponding to the $\mathrm{CP}$. Although both AcLV antisera reacted with the virus, the reactivity and specificity of the SDS-PB/ppV antisera were higher in ELISA and western blot. This confirms our previous results with antisera produced against Potato virus $Y$ and Iris yellow spot virus (6). These antisera did not react with the carlaviruses PVM and PVS in either ELISA or western blots. Furthermore, antisera against PVM and PVS reacted with the homologous virus but not with AcLV, indicating that these viruses are serologically different.

The sequenced clone included 71 nucleotides from the $5^{\prime}$ NTR and 549 nucleotides resembling part of the carlavirus ORF 1. A portion of the $5^{\prime}$ NTR is conserved among carlaviruses $(3,11,19)$, and nucleotide sequence comparison of this region showed $64.1 \%$ identity, while the amino acid sequences were $61.9 \%$ identical. The phylogenetic relationship between AcLV and other filamentous viruses, based on analysis of a small portion of the viral genome, showed that the members of each virus group clustered together as expected. Several features of the analysis indicate that PVM and AcLV are clearly distinct from other carlaviruses and that AcLV should be considered as a virus distinct from PVM. Guidelines for demarcation of virus species cannot be exactly defined and have to be established for each virus genus or family (17). Data are limited for the entire genome sequence of AcLV and other carlaviruses. However, in the Geminivirideae and Potyvirideae plant virus families, isolates with less than 85 to $90 \%$ nucleotide identity in the entire genome would normally be regarded as different viruses (17). These criteria are exceeded substantially for the AcLV-PVM comparison (64.1\%), indicating that the differences are of an order that separates distinct viruses.

In conclusion, based on particle morphology, the absence of inclusion bodies in ultrathin sections, the $M_{\mathrm{r}}$ of the $\mathrm{CP}$, and the nucleotide sequence, it is suggested that this virus belongs to the carlavirus group. Moreover, the host range, the differences in $M_{\mathrm{r}}$ of the $\mathrm{CP}$, the serological data, the nucleotide sequence, and predicted amino acid homology provide sufficient justification to consider AcLV a distinct entity and a new member of the carlavirus group. In considering a suitable designation for the A. napellus virus, we propose that it be called Aconitum latent virus (AcLV) in order to designate the natural host of the virus.

\section{ACKNOWLEDGMENTS}

Contribution from the Agricultural Research Organization, The Volcani Center, Bet Dagan, Israel, No. 512/99. The nucleotide sequence data reported in this paper will appear in GenBank under accession number AF177225.

\section{LITERATURE CITED}

1. Altschul, S. F., Gish, W., Miller, W., Myers, E. W., and Lipman, D. J. 1990. Basic local alignment search tool. J. Mol. Biol. 215:403-410.

2. Bar-Joseph, M., Gumpf, D. J., Dodds, J. A., Rosner, A., and Ginzberg, I. 1985. A simple purification method for citrus tristeza virus and estimation of its genome size. Phytopathology 75:195-198.

3. Cavileer, T. D., Halpern, B. T., Lawrence, D. M., Podleckis, E. V., Martin, R. R., and Hillman, B. I. 1994. Nucleotide sequence of the carlavirus associated with blueberry scorch and similar diseases. J. Gen. Virol. 75:711-720.

4. Clark, M. F., and Adams, A. N. 1977. Characteristics of the microplate method of enzyme-linked immunosorbent assay (ELISA) for the detection of plant viruses. J. Gen. Virol. 34:475-483. 
5. Cohen, J., Zeidan, M., Frank, A., Beckelman, H., Guttman, S., and Gera, A. 1998. Viruses in aconitum. Phytoparasitica 26:166-167.

6. Gera, A., Cohen, J., Kritzman, A., Beckelman, E., and Bar-Joseph, M. 1999. Production of diagnostic antibodies to plant viruses utilizing denatured coat proteins and booster immunization with partially purified virus. J. Plant Pathol. 81(3): 189-192.

7. Gera, A., Loebenstein, G., Salomon, R., and Franck, A. 1990. Inhibitor of virus replication from protoplasts of a hypersensitive tobacco cultivar infected with tobacco mosaic virus is associated with a $23-\mathrm{K}$ protein species. Phytopathology 80:78-81.

8. Jelkmann, W. 1994. Nucleotide sequences of apple stem pitting virus and of the coat protein gene of a similar virus from pear associated with vein yellows disease and their relationship with potex- and carlaviruses. J. Gen. Virol. 75:1535-1542.

9. Meng, B., Pang, S. Z., Forsline, P. L., McFerson, J. R., and Gonsalves, D. 1998. Nucleotide sequence and genome structure of grapevine rupestris stem pitting associated virus-1 reveal similarities to apple stem pitting virus. J. Gen. Virol. 79:2059-2069.

10. Milne, R. G., and Luisoni, E. 1975. Rapid high-resolution immune electron microscopy of plant viruses. Virology 68:270-274.

11. Monis, J., and de Zoeten, G. A. 1990. Molecular cloning and physical mapping of potato virus S complementary DNA. Phytopathology 80: 446-450.

12. Orion, D., and Frank, A. 1990. An electron microscopy study of cell wall lysis by Meloidogyne javanica gelatinous matrix. Rev. Nematol. 13:105-107.

13. Rosner, A., Ginzberg, I., and Bar-Joseph, M. 1983. Molecular cloning of complementary DNA sequences of citrus tristeza virus RNA. J. Gen. Vi- rol. 64:1757-1763.

14. Saito, N., and Nei, M. 1987. The neighbor joining method: A new method for reconstructing phylogenetic trees. Mol. Biol. Evol. 4:406-425.

15. Sambrook, J., Fritsch, E. F., and Maniatis, T. 1989. Molecular Cloning. A Laboratory Manual. 2nd ed. Cold Spring Harbor Laboratory, Cold Spring Harbor, NY.

16. Towbin, H., Staehelin, T., and Gordon, J. 1979. Electrophoretic transfer of proteins from polyacrylamide gels to nitrocellulose sheets: Procedure and some applications. Proc. Natl. Acad. Sci. U.S.A. 76:4350-4354.

17. Van Regenmortel, M. H. V., Bishop, D. H. L., Fauquet, C. M., Mayo, M. A., Maniloff, J., and Calisher, C. H. 1997. Guidelines to the demarcation of virus species. Arch. Virol. 142:1505-1518.

18. Wetter, C., and Milne, R. G. 1981. Carlaviruses. Pages 696-730 in: Handbook of Plant Virus Infections and Comparative Diagnosis. E. Kurstak, ed. Elsevier/North-Holland Biomedical Press, Amsterdam, the Netherlands.

19. Zavriev, S. K., Kanyuka, K. V., and Levay, K. E. 1991. The genome organization of potato virus M RNA. J. Gen. Virol. 72:9-14.

20. Zeidan, M., Cohen, J., Watad, A., and Gera, A. 1998. Improved purification and molecular properties of Ornithogalum mosaic virus in Israel. Ann. Appl. Biol. 133:167-176.

21. Zhang, Y. P., Kirkpatrick, B. C., Smart, C. D., and Uyemoto, J. K. 1998. cDNA cloning and molecular characterization of cherry green ring mottle virus. J. Gen. Virol. 79:2275-2281.

22. Zhang, Y.-P., Uyemoto, J. K., Golino, D. A., and Rowhani, A. 1998. Nucleotide sequence and RT-PCR detection of a virus associated with grapevine rupestris stem-pitting disease. Phytopathology 88:12311237. 